60-61| 2018

La culture dans l'enseignement du français langue étrangère: conceptions théoriques, programmes et manuels auX $\mathrm{XIX}^{\mathrm{e}}$ et $\mathrm{XX}^{\mathrm{e}}$ siècles

\title{
La dimension culturelle dans les manuels illustrés pour enfants
}

Le cas de La collection enfantine de Théodore Kyprios:

The cultural dimension in illustrated children's textbooks: The case of Theodore Kyprios' Children's Collection

Despina Provata et Marina Vihou

\section{OpenEdition}

\section{Journals}

Édition électronique

URL : https://journals.openedition.org/dhfles/5704

DOI : $10.4000 /$ dhfles.5704

ISSN : 2221-4038

Éditeur

Société Internationale pour l'Histoire du Français Langue Étrangère ou Seconde

Édition imprimée

Date de publication : 1 janvier 2018

ISSN : 0992-7654

Référence électronique

Despina Provata et Marina Vihou, "La dimension culturelle dans les manuels illustrés pour enfants », Documents pour l'histoire du français langue étrangère ou seconde [En ligne], 60-61 | 2018, mis en ligne le 03 juillet 2019, consulté le 25 mars 2023. URL : http://journals.openedition.org/dhfles/5704 ; DOI : https://doi.org/10.4000/dhfles.5704

Ce document a été généré automatiquement le 25 mars 2023.

Tous droits réservés 


\title{
La dimension culturelle dans les manuels illustrés pour enfants
}

\author{
Le cas de La collection enfantine de Théodore Kyprios: \\ The cultural dimension in illustrated children's textbooks: The case of Theodore \\ Kyprios' Children's Collection
}

Despina Provata et Marina Vihou

1 La première moitié du XXe siècle est pour la Grèce une période de profondes mutations ponctuée par deux grandes réformes éducatives révélatrices des conflits idéologiques qui, dans le domaine de l'enseignement, opposent d'un côté les traditionnalistes, partisans de la katharevousa, la langue pure, et de l'autre les modernistes qui sont pour le démotique, la langue populaire. Dès 1917, l'adoption du démotique dans les premières classes de l'enseignement primaire ${ }^{1}$ déclenche une modernisation des contenus de l'enseignement qui culminera en 1929 avec une refonte considérable de la structure même de l'école modifiant ainsi l'ensemble du système scolaire ${ }^{2}$. Ces deux réformes visaient à réorienter l'enseignement vers les réalités du temps, à l'adapter aux nouveaux besoins de la société et à faire de l'enseignement un facteur de progrès économique, sans toutefois abandonner le principe d'une école centrée sur les idéaux de l'hellénisme. En effet, toutes les disciplines, même les langues étrangères étaient mises à profit afin de consolider le sentiment national.

2 Dans ce cadre, l'État grec décide de réglementer le statut des écoles étrangères, dont celles françaises, et de vérifier l'application des lois les concernant. Ces établissements étaient en effet accusés d'éloigner les élèves grecs des valeurs patriotiques et de cultiver l'idée que les cultures des pays européens étaient supérieures à leur propre culture. Dans une circulaire du ministère des Affaires étrangères du 14 octobre 1930, rédigée à l'attention des Ambassades grecques, on peut lire à ce propos :

Ces écoles ne se souciaient aucunement que les jeunes Grecs acquièrent une formation globale conforme à l'esprit national. Au contraire, elles soulignaient plutôt les mérites de la civilisation de l'État duquel elle dépendait, ce qui signifie que les élèves étaient imprégnés des idées d'un environnement étranger et éprouvaient pour ainsi dire du mépris à l'égard de leur propre patrie qu'ils 
considéraient comme inférieure dans le domaine de la culture comparativement à

la culture des grands États occidentaux. (Antoniou 2011 : 115)

C'est suite à ce raisonnement que la loi dite Papandréou adoptée en 1931 interdit aux enfants de nationalité hellénique, fussent-ils de rite catholique, de fréquenter les écoles étrangères d'enseignement primaire (Loi 4862, $\mathrm{JO}^{\circ} \mathrm{n}^{\circ}$ 2/A, 07-01-1931). L'objectif était non seulement d'organiser un enseignement homogène pour tous les jeunes Grecs mais aussi d'édifier une nouvelle Nation. Il va sans dire que cette décision a jeté un froid dans les relations franco-helléniques. Si plusieurs écoles étrangères ont été dès lors condamnées au marasme, les élèves grecs conservaient cependant la possibilité d'apprendre le français dans d'autres établissements qui fonctionnaient librement en Grèce, comme l'Alliance Française ${ }^{3}$ ou des instituts privés de langue, comme celui fondé et dirigé par Théodore Kyprios, l'auteur de la Collection enfantine qui nous intéresse pour cette étude.

Cette méthode, sans doute la première à avoir été illustrée à l'intention des élèves du primaire, apparaît pour répondre aux besoins spécifiques de cette nouvelle clientèle. Mais avant de nous pencher sur cette collection de manuels et notamment sur la manière dont l'image se met au service de l'enseignement de la culture française, nous en examinerons le contexte institutionnel, à savoir le cadre législatif et les dispositions officielles qui définissent la politique éducative et les objectifs pédagogiques de l'enseignement du français en Grèce.

\section{Les images dans les manuels scolaires de français en Grèce}

5 À partir de la première décennie $\mathrm{du} \mathrm{XX}^{\mathrm{e}}$ siècle les voix qui militent en Grèce en faveur de l'introduction des nouvelles pratiques pédagogiques dans l'enseignement des langues, gagnent progressivement du terrain. La méthode traditionnelle recule au profit d'une approche intuitive de la langue et de l'enseignement par l'aspect, initié par des pédagogues Allemands qui, dans le cadre des leçons de choses, se servaient des séries de tableaux muraux (Reinfried 1993 : 3-4). C'est ainsi que l'image prend peu à peu sa place dans les manuels de français, avec un grand retard toutefois par rapport à ce que l'on a pu observer dans d'autres pays européens (Reinfried 1993 ; Van der Sanden 2012).

6 En effet, ce n'est qu'en 1907 que les images et notamment celles de l'imprimeur autrichien Eduard Hölzel deviennent officiellement le support de l'enseignement pour le français (JO, n 139/12-07-1907). L'État grec lance alors un concours pour la rédaction de nouveaux manuels scolaires pour le français et fournit aux auteurs des instructions précises $^{4}$. Le manuel conçu pour l'École hellénique ${ }^{5}$ devait présenter « les objets de la vie de l'élève (dans l'école, la famille, la nature) et l'image de la ville telle qu'elle figure sur les tableaux de Hölzel, ainsi que des passages de descriptions et de lectures faciles qui se réfèrent à la vie des élèves en général et la nature environnante ». Pour les classes du Gymnase, le manuel devait s'appuyer également sur les images de Hölzel du printemps, de l'été, de l'automne, de l'hiver, de la ferme, du port, et de l'appartement parisien (ibid.). Si l'enseignement par l'aspect trouve désormais sa place dans l'enseignement du français en Grèce, la méthode traditionnelle n'est toutefois pas abandonnée: la grammaire et la traduction (version et thème) constituent toujours le couronnement de tout enseignement du français pour les classes supérieures. 
7 Deux ans plus tard, en 1909, paraissent les premiers manuels approuvés pour l'enseignement public inspirés de la méthode directe : un pour l'École Hellénique, de Gérasime Perrobonis, et un pour le Gymnase, de Antonios Syrigos ${ }^{6}$. Malgré le recul évident du texte littéraire dans l'enseignement de la langue au profit du texte nonlittéraire et de la leçon de choses, la présence des images et leur exploitation reste encore limitée. Ces deux manuels, qui sont édités sous la forme d'un cours complet, contiennent de la grammaire, des textes à lire, des morceaux à traduire tandis que les consignes aux élèves sont données en grec. Même si l'image sert à stimuler la compréhension de l'élève et à déclencher sa participation orale au cours, à l'instar de l'enseignement par l'aspect (Reinfried 1999: 4-5), les textes continuent d'avoir une existence autonome.

8 Le programme scolaire de 1914 maintient les mêmes dispositions quant à l'utilisation des images de Hölzel et l'exploitation du texte littéraire choisi. Mais on y lit deux nouveaux commentaires: il est recommandé d'utiliser le plus possible la langue française pendant le cours, ce qui entraîne pour la première fois un recul de la langue maternelle dans les classes tandis que les morceaux littéraires choisis doivent « susciter autant que possible l'intérêt national du jeune Hellène » (JO, nº 343/A, 24-11-1914). Le souci de mettre la langue française à contribution pour renforcer la conscience nationale, qui caractérisait déjà les chrestomathies françaises du XIX siècle, se maintient (Provata 2011).

9 Cependant, commence à s'amorcer un tournant vers l'enseignement de la civilisation : il ne suffit plus de découvrir les particularités d'un peuple à travers sa langue et sa littérature, il faut aussi le connaître dans sa quotidienneté. Ainsi, le ministère demande que l'image de la ville de Paris soit exploitée dans les classes de langue (ibid.).

Cette approche interculturelle se précise davantage dans le programme de 1932 qui concerne le nouveau Gymnase de six ans. Alors que pendant les deux premières années de français l'enseignement se focalise exclusivement sur l'apprentissage de la langue à travers des textes qui évoquent l'école et la vie scolaire, la vie quotidienne et le corps humain, un apprentissage qui est en outre complété par la grammaire et la traduction (thème et version), la troisième année de français prévoit l'enseignement de la civilisation. Les textes et les images qui évoquent la vie urbaine (vie économique et culturelle, les transports) ainsi que la vie à la campagne, ne sont plus vagues mais dorénavant "peuvent être situés en France", c'est-à-dire avoir comme cadre un environnement culturel concret. Quant à la littérature française classique et moderne, elle doit aussi servir à "démontrer l'influence de la littérature française sur la littérature néo-hellénique ". Il s'agit là, avant l'heure, d'une approche comparative de la littérature dans les Gymnases alors que la discipline n'est pas encore constituée en Grèce! Mais l'on va encore plus loin, car l'étude des textes littéraires se proposait de repérer «les éléments de la civilisation française moderne et de son influence sur la civilisation contemporaine " (JO, $\left.\mathrm{n}^{\circ} 12 / \mathrm{A}, 13-1-1932\right)$. Ces dispositions assez novatrices se maintiennent aussi lorsque l'on révise le programme scolaire en 1935. On insiste alors sur la nécessité de faire apprécier aux élèves non seulement les textes littéraires mais aussi d'autres éléments de la civilisation française. Ici aussi c'est à partir de la troisième année de français qu'est introduite l'approche civilisationnelle de la langue (JO, n 537/A, 9-11-1935). 


\section{La Collection enfantine de Théodore Kyprios}

11 Tout en tenant compte des dispositions des textes officiels pour l'enseignement secondaire, la collection enfantine de Théodore Kyprios propose une approche de la langue et de la culture françaises adaptée aux besoins spécifiques des jeunes apprenants, probablement des classes supérieures du primaire.

Peu de choses sont connues sur le parcours professionnel de Kyprios, ou Kyriakopoulos de son vrai nom, né en 1887, qui est l'auteur de manuels pour français le plus polygraphe de son temps : il a publié des grammaires, des dictionnaires bilingues (grecfrançais et français-grec), des manuels destinés aux établissements secondaires mais aussi des manuels pour l'auto-apprentissage $d u$ français, dialogues familiers, chrestomathies et anthologies, des manuels de français spécialement conçus pour les écoles de commerce, sans oublier la publication d'un périodique destiné à l'autoapprentissage du français, le Journal français, l'unique en son genre dans le pays (Provata 2017). Il est aussi parmi les premiers à reconnaître l'importance de la méthode directe pour apprendre une langue étrangère et organise, à partir de 1908, des cours de pratique orale fondés sur les images de Hölzel (Journal français, $\mathrm{n}^{\circ} 23,23$ août $1908: 4$ ).

La collection de manuels que Kyprios compile pour les classes enfantines est importante. Elle est constituée de deux syllabaires comportant chacun des gravures originales (69 et 76 gravures réciproquement); d'un petit vocabulaire illustré qui fait suite aux syllabaires et qui comporte aussi 18 tableaux-gravures ; un manuel intitulé Le français par l'image avec 37 gravures originales; un livre auxiliaire Les Quatre saisons d'après Hölzel avec une réduction des quatre tableaux muraux et 30 gravures dans le texte; un livre élémentaire de lecture et d'élocution intitulé Mon livre d'histoires comportant 30 gravures originales; un cours élémentaire de grammaire française au nom de Ma petite grammaire; un Livre d'anecdotes. Enfin, un livre de lecture courante intitulé Paris couronne le tout avec 32 reproductions photographiques et un plan de Paris hors texte.

Il est difficile de se prononcer avec certitude sur la date de publication de cette collection de manuels, la seule indication chronologique que l'on possède étant la date d'entrée dans les fonds de la Bibliothèque nationale de Grèce en 1939. Vu les dispositions du dépôt légal, cette date nous permet de la considérer comme étant proche de la date de parution. On sait par ailleurs que cette collection ne faisait pas partie du grand projet de publications pédagogiques, intitulé « Bibliothèque française ", qui ambitionnait d'être un " système complet » pour l'étude du français, et que Kyprios avait fièrement annoncé en 1910 dans les pages du journal qu'il publiait (Journal français, 18 septembre $1910: 72$ ). On peut également supposer un probable terminus post quem, ou date butoir la plus ancienne, celle de 1931. En effet, à cette date la réforme du livre scolaire divise les manuels en trois catégories selon leur but et leur fonction : les manuels proprement dits, les ouvrages auxiliaires et les lectures libres incitant ainsi, aussi bien le professeur que l'élève, à recourir à d'autres manuels, parallèles au manuel proprement dit (JO, $\mathrm{n}^{\circ}$ 165/A, 23-6-1931). Signalons également que la rareté de ce manuel - il n'en existe qu'un seul exemplaire dans les grandes bibliothèques d'Athènes alors qu'il en est à sa $3^{\mathrm{e}}$ édition - renforce l'hypothèse qu'il était exclusivement destiné à l'enseignement du français dans des instituts de langue privés, suite à la loi de 1931, voire à un usage domestique. 
15 S'il est difficile de définir avec certitude le public auquel s'adressait cette méthode ou de mesurer son impact dans l'enseignement de la langue française, il s'avère encore plus difficile d'en identifier le ou les illustrateurs. Kyprios parle de "gravures originales" mais seule une recherche plus approfondie sur les illustrateurs des manuels grecs de cette période pourrait fournir une réponse à cette question. Une signature "Kotik» que portent certaines images dans Mon livre d'histoires et qu'il ne nous a pas été possible d'identifier, ne nous permet pas de conclure sur la question.

\section{Le français par l'image}

16 Le titre même du manuel Le français par l'image souligne le rôle prépondérant que son concepteur réserve à l'image dans l'enseignement de la langue française. Il s'agit sans aucun doute d'une innovation pédagogique et didactique de l'époque que Kyprios entendait souligner : l'image n'est plus accessoire mais devient moyen d'enseignement à une époque où, dans les manuels de français utilisés en milieu scolaire, l'image continue à avoir un rôle décoratif.

En tant que moyen d'enseignement, l'image visait surtout à susciter l'intérêt de l'élève mais elle permettait aussi à l'enseignant de faire entrer par la méthode intuitive la représentation des objets du monde. Mais quel rôle Kyprios entend-il attribuer à l'image ? Dans quelle mesure ces images font-elles la différence entre la culture locale, familière et proche de l'élève, et la culture-cible? Quelle est finalement la charge culturelle que véhiculent les images dans ses manuels?

18 Le recours à l'image visait à la mobilisation des trois types de l'intuition, tels qu'ils avaient déjà été définis par Ferdinand Buisson en 1878 : «l'intuition sensible, celle qui se fait par les sens; l'intuition mentale proprement dite, celle qui s'exerce par le jugement sans l'intermédiaire de phénomènes sensibles ou de démonstration en règle ; enfin l'intuition morale, celle qui s'adresse au cœur et à la conscience » (1880:325-368). S'appuyant sur cette fonction fondamentale de l'image, Le Français par l'image va encore plus loin et réussit à renverser le processus même d'appréhension de la langue étrangère: au lieu d'aller du signifiant vers le signifié (ce qui est le cas de l'enseignement du vocabulaire), on va du signifié au signifiant (ce qui est le cas des images du manuel de Kyprios). En avançant la représentation iconique d'une "réalité » schématisée et en soumettant le contenu d'un texte à la description précise d'une image, son appréhension mobilise l'intuition sensible de l'élève, mais aussi son intuition mentale (par exemple, pour les phénomènes grammaticaux) et morale, pour les messages implicites véhiculés.

19 En outre, la représentation iconique de la vie quotidienne dans les manuels de Kyprios vise à créer des rapports visuels entre la réalité des élèves et celle de la langue étudiée et à imiter ainsi les méthodes naturelle et directe de l'acquisition de la langue maternelle.

20 Articulé autour de 32 leçons, le manuel est réparti en six catégories thématiques : a) l'école, b) la maison familiale, c) la ville et les moyens de transports, d) les boutiques des commerçants, e) le village et la campagne, f) les saisons de l'année. Le titre de chaque leçon, le nouveau vocabulaire ainsi que les exercices d'entraînement et de réutilisation sont étroitement liés à une image qui constitue d'ailleurs le point de 
départ de chaque unité didactique. Cette structuration de la leçon mobilise les opérations cognitives des élèves, telles que l'observation et la réflexion critique.

Destinées à un public de jeunes apprenants, les images présentes dans le manuel sont simples dans leur conception et restent dans leur majorité des images dépourvues de tout élément périphérique risquant de perturber la compréhension. Les personnages présents, avec l'élève au centre de son milieu quotidien, à savoir la famille, le maitre et les camarades de classe, n'évoquent aucune caractéristique émotionnelle. Ils font eux aussi partie du «paysage» représenté et sont démunis de toute profondeur psychologique. La simplicité iconographique et la naïveté esthétique entendaient vraisemblablement assurer l'intelligibilité de l'image de la part d'un public de jeunes élèves débutants dans l'apprentissage de la langue française. Kyprios obéit donc à cette règle primordiale: la simplicité des dessins et l'absence de détails "bavards" favoriseraient la compréhension par les petits élèves et lèveraient toute ambiguïté.

Chaque unité du manuel Le français par l'image est composée de quatre rubriques disposées verticalement l'une après l'autre sur la même page. Cette disposition verticale suit une certaine progression linéaire, partant de la représentation visuelle d'une situation familière vers la réutilisation des éléments appris, tout en passant par l'explicitation détaillée de l'image. Chaque image illustrant des petites scènes de la vie quotidienne de l'élève, est suivie du nouveau vocabulaire. La compréhension des mots est ainsi facilitée par leur visualisation. Le maître, faisant complètement abstraction de la traduction, se limite à aider l'élève à associer directement et intuitivement les objets et les personnages de l'image au "Vocabulaire». La troisième rubrique, intitulée « Description de l'image », décrit de façon simple et linéaire les images en reprenant les mots du vocabulaire, ce qui souligne la complémentarité étroite entre l'image et le petit texte descriptif. Enfin, après avoir incité les élèves à lire et à comprendre la langue parlée, les « exercices de langage " les incitent à répondre à des questions sur le texte. C'est le moment de la fixation des nouvelles connaissances mais aussi de leur réutilisation plus ou moins spontanée.

Les thèmes des leçons du manuel sont focalisés, comme nous l'avons dit, sur la civilisation quotidienne. L'école, la famille, le marché, la ville et le village constituent autant d'images de la culture partagée de l'époque. Éloignés donc de la civilisation savante, les contenus du manuel témoignent de son caractère utilitaire et pratique et d'une conception de l'enseignement des réalités. Une réalité qui demeure toutefois ambiguë dans les pages du manuel. 


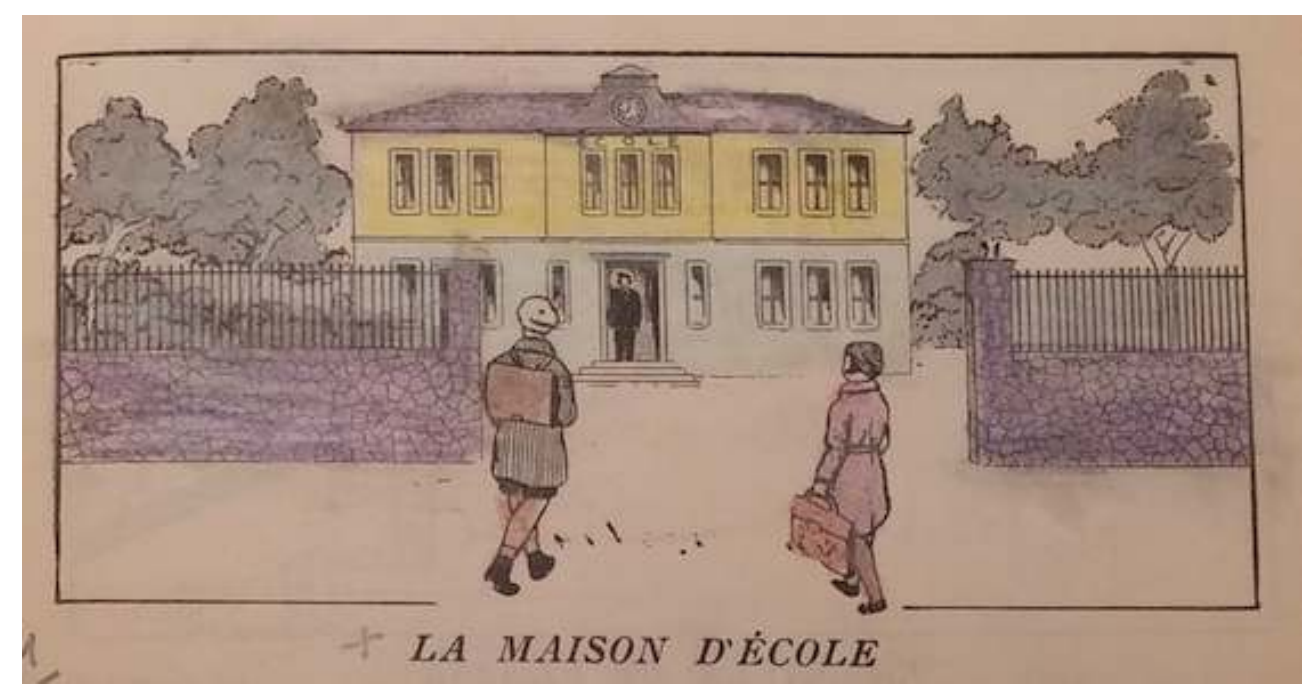

C'est l'image de «la maison d'école » qui ouvre l'ensemble des leçons du manuel, un bâtiment qui assumait en France une double fonction: logement du maître et salles organisées et outillées pour recevoir les élèves (Blondel 1911).

Ce bâtiment emblématique de la Troisième République, érigé dans le style d'une architecture standardisée qui avait doté la France d'écoles à deux étages, aux fenêtres larges et nombreuses, avec à l'étage le logement du maître, l'inscription sur la façade et la grande horloge ronde qui renvoie à un édifice public prestigieux, sans oublier la cour de récréation sur le devant, avec jardin et lieux d'aisance, est sans aucun doute étranger à l'élève grec. Car, bâties selon les caractéristiques du courant architectural néoclassique, les écoles grecques construites à la fin du XIX ${ }^{\mathrm{e}}$ et au début du XX $\mathrm{XX}^{\mathrm{e}}$ siècle se déploient sur le rez-de-chaussée et ont une cour intérieure invisible depuis la façade du bâtiment alors que dans des régions plus pauvres, les bâtiments scolaires sont souvent des constructions simples, rectangulaires et sans cour.

Si cette première approche du milieu scolaire tente de familiariser l'élève avec la culture cible, la représentation de la salle de cours vise plutôt à l'effet d'identification avec la culture locale et semble s'aligner sur les directives du ministère de l'éducation nationale qui prescrivaient que même dans le cadre des classes de français, l'élément national soit accentué. Ainsi, sur le mur de cette salle de classe mixte domine une carte de la Grèce.

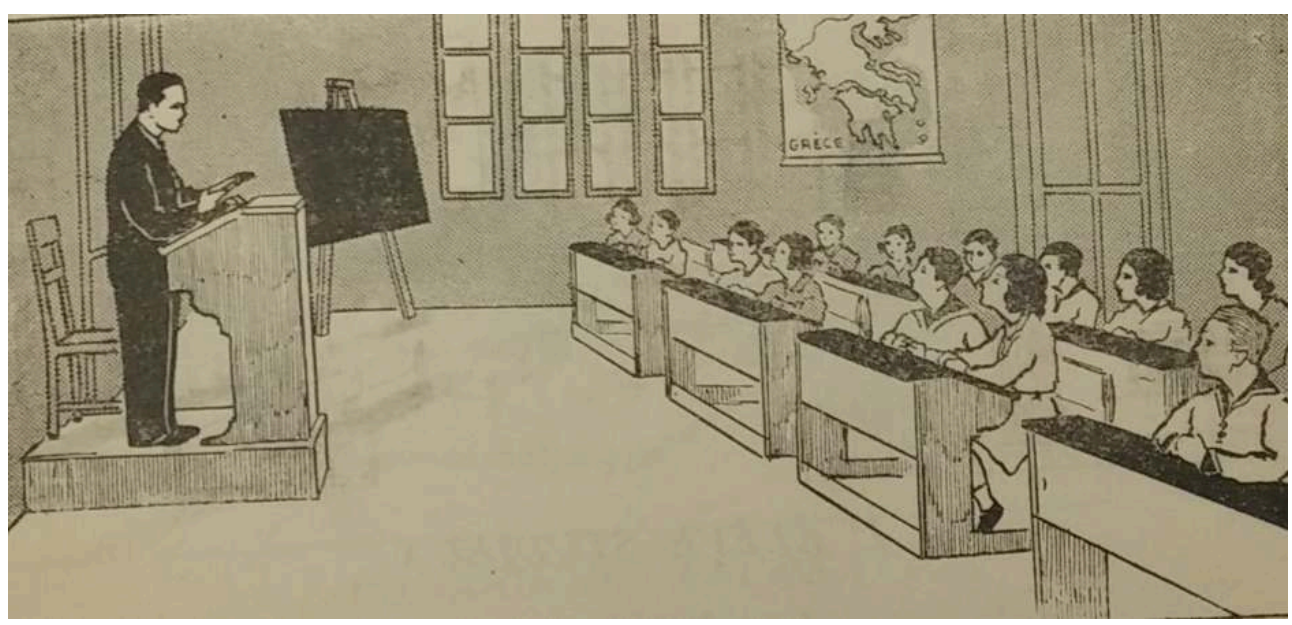



l'époque dans son intégralité : la Crète, annexée en 1913 en est absente. Omission volontaire dans un souci de simplification de l'image ou négligence du dessinateur ? On ne saurait répondre avec certitude.

Cest surtout la vie quotidienne de l'époque, celle de l'élève ou d'une manière plus générale celle de la société environnante qui occupe la plus grande partie du manuel, comme le suggéraient les programmes scolaires en vigueur. En effet, à travers les scènes évoquées, sont proposés autant de modèles comportementaux qui devaient cultiver chez l'élève des valeurs sociales telles que l'obéissance, la gentillesse, la moralité. Ainsi, la journée de l'élève se déploie du lever, avec la préparation pour aller à l'école et le rituel de l'hygiène personnelle, jusqu'au soir où la famille se rassemble dans la belle salle de séjour.

L'intérieur de la maison familiale renvoie à un milieu bourgeois, celui probablement dont sont issus ces jeunes qui ont les moyens d'apprendre le français dès un très jeune âge, bien avant celui prévu dans l'enseignement public. La famille type de la bourgeoisie est représentée ici dans un environnement confortable et offre des modèles à suivre et à imiter : l'élève studieux en train de faire ses devoirs, la petite fille modèle qui joue du piano, aide aux travaux ménagers et fait son apprentissage aux travaux à l'aiguille auprès de sa mère. L'image de la mère offre aussi un modèle stéréotypé de la bourgeoise qui s'occupe de son foyer et de ses enfants et se fait aider par une domestique pour les travaux ménagers. De l'autre côté, le père, en costume sombre et en train de lire son journal, distant mais physiquement présent, incarne la réussite professionnelle. Les dialogues qui accompagnent les images sont l'occasion de souligner les aspects moraux et éducatifs d'une certaine classe sociale.

Si l'on compare ce manuel aux livres utilisés pour l'enseignement du grec mais aussi à des ouvrages ayant servi pour l'enseignement du français dans d'autres pays européens (Kok Escalle \& Van Strien-Chardonneau 2007), on constate que la religion y est pratiquement absente. En effet, un crucifix placé au-dessus du lit du jeune garçon et le soupçon d'une icône toujours au-dessus de son lit lorsqu'il est représenté malade, sont les seules références à la religion. Notons au passage, que dans la tradition orthodoxe, ce sont des icônes et non le crucifix qui sont présentes dans les chambres à coucher.

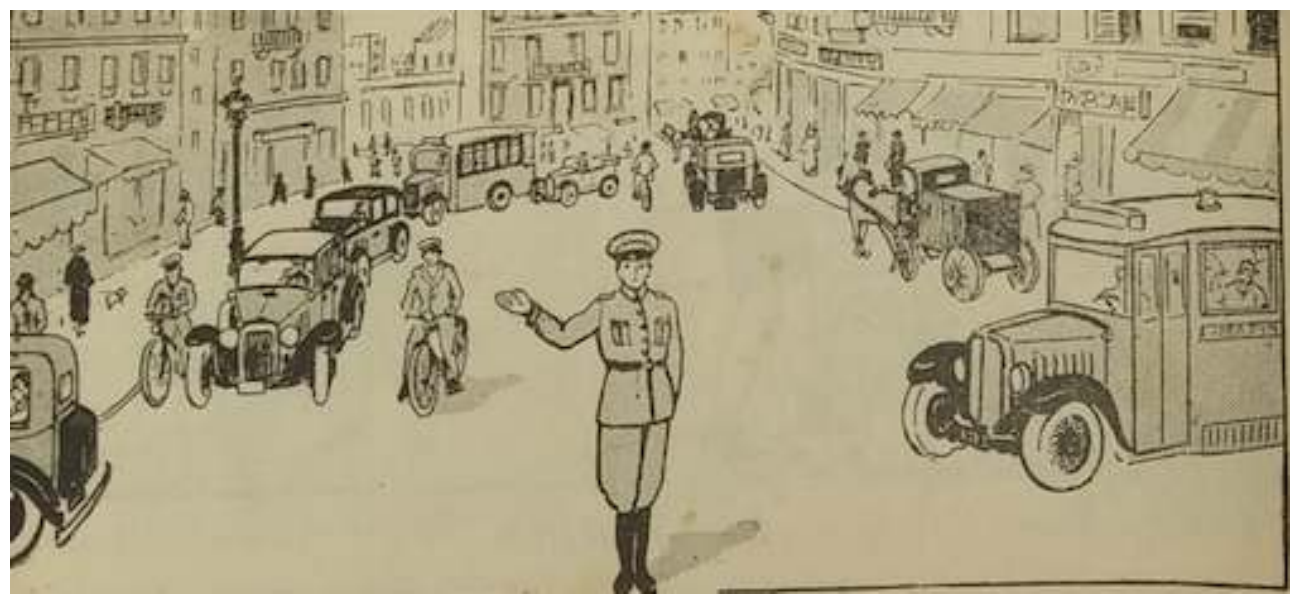

31 Selon les dispositions du programme officiel, les manuels de français devaient aussi évoquer l'environnement proche de l'élève c'est-à-dire, pour la bourgeoisie montante, la vie urbaine. Si l'identité de la ville présente sur ces images n'est pas clairement 
évoquée, la figure de l'agent de police réglant la circulation renvoie à la culture proche de l'élève ; il porte en effet l'uniforme du corps spécial grec de la police urbaine.

La ville c'est aussi l'image de la modernité, le lieu qui permet aux jeunes enfants de se familiariser avec les nouveautés technologiques de l'époque. Ainsi les images et les descriptions qui les accompagnent sur l'utilisation de l'électricité et de la vapeur dans les moyens de transport, les pratiques liées aux voyages, se présentent comme autant de connaissances techniques utiles liées à la modernisation de la ville.

Les métiers et les pratiques commerciales présentés à travers les images et les textes du manuel visent à familiariser l'enfant avec la vie quotidienne. Cependant, si d'une manière générale le vocabulaire reste simple, il arrive, comme dans le cas de la leçon sur l'atelier de menuisier, de lui proposer un vocabulaire spécifique : copeaux, établi, varlope, scie. Il ne s'agit plus ici de connaissances courantes et de vocabulaire fondamental mais de vocabulaire spécifique, qui met en avant l'idée d'un savoir spécialisé et atteste tant la perméabilité des frontières entre l'ordinaire et le spécialisé, qu'entre les classes d'une société en pleine mutation, qui fait l'expérience de l'exode rural et du passage à une urbanisation de plus en plus importante.

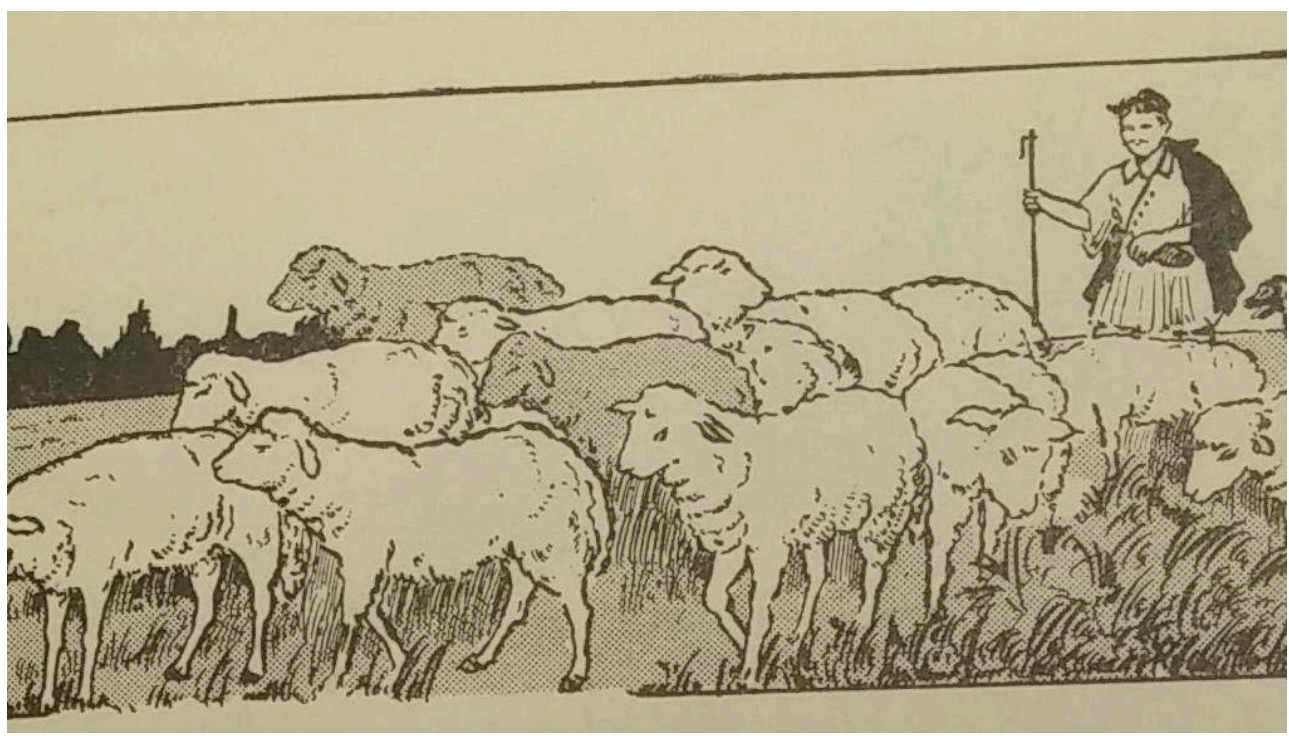

On ne saurait donc s'étonner que les dernières leçons du manuel soient consacrées à la vie au village et à la nature. Au centre de l'intérêt se trouvent l'élevage et l'agriculture. Mais alors que l'image de la ville se caractérise par - comme on l'a vu - une certaine neutralité culturelle, une identité globale dirait-on peut-être aujourd'hui, l'image du troupeau d'agneaux et du berger portant le costume traditionnel grec, la fustanelle, renvoie directement à la campagne grecque.

À l'exception donc de certaines touches aisément reconnaissables, et comme appliquées çà et là pour répondre aux exigences ministérielles, le manuel fait donc preuve d'une certaine neutralité culturelle car la culture française, reste sous-jacente. Si l'on apprend la langue de l'Autre, sa culture est d'abord seulement suggérée. L'enseignement du français offre bien un modèle à suivre, mais il s'agit toutefois moins d'un modèle étranger que de celui recherché et imposé dans l'enseignement grec visant avant tout de former de jeunes écoliers accomplis et à promouvoir les valeurs morales de l'époque, telles que la discipline, l'obéissance, l'effort et l'aspiration au progrès. 


\section{La dimension interculturelle de la Collection enfantine}

que la dimension interculturelle soit presque absente du manuel Le français par l'image, elle n'est toutefois pas exclue de la collection enfantine de Kyprios. C'est en effet aux manuels complémentaires que ce rôle est dévolu. Nous laisserons de côté les deux Syllabaires, le Vocabulaire illustré et la Grammaire, ainsi que Les Quatre saisons, dont les gravures ne sont pas "originales" pour nous concentrer sur les trois manuels complémentaires de la collection enfantine: Mon livre d'histoires, Mon livre d'anecdotes et Paris.

Dans Mon livre d'histoires, l'objectif linguistique est étroitement associé au but éducatif. Kyprios propose ici des historiettes suivies d'exercices de langue et de questions de compréhension. Le tout est couronné par des maximes et parfois de brefs poèmes. La plupart des textes sont anonymes, à l'exception de rares cas où est mentionné le nom de l'auteur : trois poèmes de Jean Richepin, deux brefs extraits de La Fontaine, un texte d'Émile Delapalme ${ }^{7}$ et de $\mathrm{P}$. J. Stahl ${ }^{8}$, et de J. Calvet ${ }^{9}$. Adressée à un très jeune public, cette petite anthologie vise uniquement à proposer aux enfants des lectures morales et édifiantes. Il s'agit d'exalter les qualités et les valeurs morales des personnages telles que la compassion, le travail, la gentillesse, le courage, la bonté et la générosité mais aussi à signaler des comportements à éviter comme l'imprudence, le mensonge, la cupidité, la convoitise ou le vol. Ce petit « livre élémentaire de lecture et d'élocution » est également accompagné de gravures originales. Or parmi ces historiettes, une seule évoque explicitement la France: il s'agit d'un extrait du Magasin Pittoresque qui rapporte une croyance bretonne selon laquelle le chant du coucou porte bonheur. Dans l'ensemble, l'iconographie occupe un rôle accessoire et ces lectures moralisantes et éducatives ne se situent pas explicitement dans un milieu français, même si la culture française est présente dans certaines représentations iconographiques (le berger ou la maison du village par exemple).

Si ces lectures moralisantes et éducatives ont très peu de rapport avec la culture française, l'approche est différente pour le Livre d'anecdotes. Destiné vraisemblablement à des élèves plus avancés dans l'étude de la langue, ce petit livre offre des lectures éducatives qui permettent aux jeunes grecs de découvrir des personnalités historiques mais aussi des écrivains français à travers des anecdotes sur leur vie. Or, les grands hommes qui doivent servir d'exemple à la jeunesse hellénique sont aussi bien grecs que français, voire européens. La lecture s'ouvre sur le portrait d'un vaillant soldat de la bataille de Marathon, se poursuit avec l'ambition de Thémistocle et les compagnons de Léonidas, s'arrête à l'épisode du nœud Gordien et au dialogue entre Alexandre le Grand et Diogène. Le souci de mettre l'enseignement du français à contribution pour créer une conscience nationale est ici évident. Outre la valeur moralisante et édifiante dont sont dotées ces historiettes, elles proposent aux élèves grecs des lectures sur des épisodes célèbres de l'histoire de France mais aussi des anecdotes sur la vie d'écrivains célèbres comme Molière, Boileau, Beaumarchais ou Dumas. C'est également l'occasion pour les élèves de lire des extraits même brefs d'auteurs célèbres tels que le duc de Saint-Simon, Voltaire, Florian, Sainte-Beuve... mais aussi Tolstoï. Dans le Livre d'anecdotes l'illustration recule au profit du texte. En effet, seules les effigies de certains personnages historiques, comme Napoléon Bonaparte, ou d'auteurs français célèbres y sont présentes. 
39 La formation générale des élèves est enfin complétée par le petit livre intitulé Paris qui leur permet de découvrir la France et la civilisation française à travers 32 reproductions photographiques et les textes qui les accompagnent. Véritable petit guide de la capitale française, le livret dresse une image prestigieuse de la ville à travers la présentation de ses monuments célèbres, comme l'Hôtel de Ville, le pont Alexandre III, le Louvre, l'Arc de Triomphe et le Petit Palais. Il présente également les conditions de vie du peuple dont on étudie la langue, notamment des aspects de la vie commerciale, économique et culturelle de la ville, dont on souligne l'ambiance moderne et animée, perpétuant le glorieux symbole d'une capitale internationale, la ville-lumière : «sur ces boulevards, la circulation est incessante pendant le jour, et jusqu'à minuit, cette longue voie est encore animée, à la lueur de milliers de cathédrales versant à flots les lumières de l'électricité le long des magasins de toute sorte, splendidement éclairés » (Paris [1939] : 13).

40 Le seul texte signé que l'on rencontre dans ce livret, une description de Paris rédigée par le journaliste Lucien Anatole Prévost Paradol, cherche à relier le passé prestigieux du Second Empire et des siècles précédents à l'actualité de la capitale française et à souligner le fait que la gloire du passé côtoie avec grâce le présent et la quotidienneté d'un peuple intelligent et séduisant. En dépit de sa grandeur, de sa taille, sa variété et ses particularités, Paris, écrit-il «n'a rien d'accablant [...], il est harmonieux [...] admirablement adapté, enfin, à la race ingénieuse, sensible et légère qui l'habite » (Paris [1939] : 8-9).

41 Cette présentation de Paris est aussi l'occasion de faire un rapprochement entre les deux civilisations. Ainsi on signale les redevances de l'église de la Madeleine au temple de Jupiter à Athènes et on ne manque pas de parler des deux chefs-d'œuvre de l'antiquité grecque conservés au Louvre, la Victoire de Samothrace et la Vénus de Milo. Ce petit livre est donc entièrement consacré à la civilisation française et à son image de marque. Ce qui le distingue des autres manuels de cette collection, c'est le souci de son concepteur de relier le passé au présent et l'histoire à la modernité de la ville de Paris.

\section{Conclusion}

Ces réflexions sur quelques images représentatives du manuel, nous permettent de formuler certaines constatations. La série d'ouvrages que Kyprios rédige à l'intention d'un public de jeunes adolescents hors du milieu scolaire ou avant l'âge institutionnel pour l'apprentissage du français, propose une nouvelle approche de l'enseignement de la langue et se veut résolument novatrice. Tout en tenant compte des instructions officielles qui étaient en vigueur en Grèce, Kyprios propose une méthode d'apprentissage par l'image associée à une série de manuels complémentaires dont chacun répond à un besoin éducatif précis. Si nous ne sommes pas en mesure d'estimer l'impact ou l'ampleur de la diffusion de cette méthode, nous pouvons en revanche affirmer que nous nous trouvons en présence d'une véritable méthode illustrée. Nous posons encore, quoiqu'avec prudence car la recherche reste encore ouverte, qu'il s'agit peut-être de la première méthode illustrée en Grèce, pour accorder une place prépondérante à l'image, dont la fonction multiple s'identifie à une ressource rhétorique. Les images ne sont plus décoratives, elles ne se limitent pas à une simple fonction esthétique, comme c'était le cas des manuels utilisés en milieu scolaire en Grèce. Dans la méthode de Kyprios, les images sont le point de départ des activités 
proposées en classe. Le monde représenté se situe à mi-chemin entre la réalité et les représentations de ces concepteurs, l'auteur et le dessinateur. Ce monde, visiblement ancré sur le présent, en présente néanmoins une version idéologiquement édulcorée qui correspond au modèle pédagogique que s'efforçait de bâtir l'État grec de l'entredeux-guerres pour qui l'école apparaissait comme le moyen de réussite et de promotion sociale. Le Français par l'image, qui sert à la première approche de la langue, se dote ainsi d'un pouvoir formateur évident, conforme aux idéaux de l'hellénisme et de la bourgeoisie montante. Si la culture française reste sous-jacente dans un premier temps, elle s'affirme progressivement à travers les lectures, les brèves biographies ou les anecdotes sur des personnages célèbres et culmine avec le livret intitulé Paris, véritable petit guide de la capitale française. Invitation à découvrir la culture et la civilisation de l'Autre, pour cette génération de Grecs francophones qui se dirigent pendant l'entredeux-guerres vers les universités françaises, ce livre est aussi une invitation au voyage, par le texte comme par l'image. Ce n'est peut-être pas un hasard si l'une des photos qui ornent ses pages est la salle Labrouste de la Bibliothèque nationale de France.

Ma petite Grammaire. Cours élémentaire de Grammaire française, à l'usage des classes enfantines. Cartonné. Athènes : s.é. pratique de la langue française à la base des images d'Ed. Hölzel]. Athènes: Impr. Sakellariou. 


\section{Sources primaires}

KYPRIOS, Théodore Collection enfantine [1939] :

Mon Syllabaire Illustré. $1^{\mathrm{er}}$ Livret, 69 gravures originales. Cartonné. Athènes : s.é.

Mon Syllabaire Illustré. $2^{\mathrm{e}}$ Livret, 76 gravures originales. Cartonné. Athènes : s.é.

Mon Petit Vocabulaire illustré. Ouvrage faisant suite aux Syllabaires. 18 tableaux-gravures.

Cartonné. Athènes : s.é.

Le Français par l'image. 37 gravures originales. Cartonné. Athènes : s.é.

Les Quatre Saisons. Le Printemps, L'Été, l'Automne, l'Hiver. Description des 4 tableaux de Hölzel, avec une réduction des 4 tableaux muraux hors texte, et 30 gravures dans le texte. Cartonné.

Athènes : s.é.

Mon Livre d'Histoires. Livre élémentaire de lecture et d'élocution, à l'usage des classes enfantines. 30 gravures originales. Cartonné. Athènes : s.é.

Mon Livre d'anecdotes. Livre élémentaire de lecture et d'élocution. Anecdotes, Historiettes, Mots Historiques. Athènes : s.é.

Paris. Livre de lecture courante. 32 reproductions photographiques un plan de Paris hors texte. Athènes : s.é.

\section{Sources secondaires}

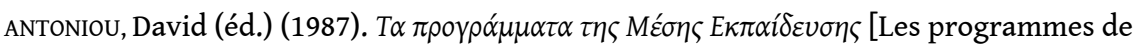
l'enseignement secondaire] (1933-1929). Athènes : IAEN.

ANTONIOU, David (2011). Écoles françaises en Grèce. Essai d'inventaire. Athènes : CIREL.

BLONDEL, charles (1911). « Maison d'École », paru dans le Nouveau Dictionnaire de Pédagogie et d'Instruction primaire, (dir. Ferdinand Buisson). En ligne :

http://www.inrp.fr/edition-electronique/lodel/dictionnaire-ferdinandbuisson/document.php? id=3100\&format=print

BUISSON, Ferdinand ( $\left.{ }^{3} 1880\right)$ [1878]. « Conférence sur l'enseignement intuitif ». In Les conférences pédagogiques faites aux instituteurs délégués à l'Exposition universelle de 1878. Paris : C. Delagrave.

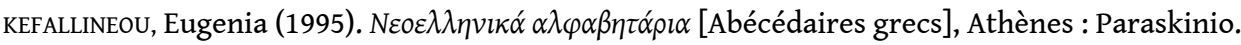
KOK ESCALLE, Marie-Christine \& VAN STRIEN-CHARDONNEAU, Madeleine (2007). « Aspects culturels et interculturels des manuels d'apprentissage du français dans les Pays Bas des XVI ${ }^{\mathrm{e}}-\mathrm{XIX}^{\mathrm{e}}$ siècles ». In Monique Lebrun (dir.), Le manuel scolaire d'ici et d'ailleurs, d'hier à demain. Québec: Presses de l'Université du Québec, 20 p. (sur CD).

KOULOURI Christina \& VENTURAS Ekaterini (1993). « Les manuels scolaires dans l'État grec, 1834-1937. Histoire de l'éducation, Manuels scolaires, États et sociétés. XIX ${ }^{e}-X X^{e}$ siècles, 58, 9-26. En 
ligne : [http://www.persee.fr/web/revues/home/prescript/article/

hedu_0221-6280_1993_num_58_1_2657] (consulté le 2 avril 2017).

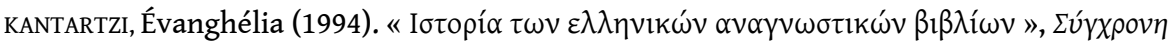

Eк $\alpha \alpha i ́ \delta \varepsilon v \sigma \eta$ [Éducation contemporaine], 75, 30-34.

PROVATA, Despina (2011). « Construction identitaire et enseignement du français en Grèce au XIX ${ }^{\mathrm{e}}$ siècle ", In Konstantinos Dimadis (dir.), Identities in the Greek world (from 1204 to the present day), Athens : European Society of Modern Greek Studies, vol. 5, 281-192.

PROVATA, Despina (2017). «Innovation et autoapprentissage par voie de presse : $H$ Г $\alpha \lambda \lambda_{l \kappa} \eta \dot{~ E \varphi \eta \mu \varepsilon p i ́ \varsigma ~}$ [Le Journal français] (1908-1911). Documents pour l'histoire du français langue étrangère ou seconde, 58-59, 103-120.

VAN DER SANDEN, Nathalie (2012). « Le rôle des images dans les ouvrages pour l'enseignement du français aux Pays-Bas (1800-1950) : statuts, fonctions et enjeux didactiques ». Documents pour l'histoire du français langue étrangère ou seconde, 49, 31-51.

REINFREID, Marcus (1993). « Et l'image vint. Le mouvement réformiste du XIXe siècle en Allemagne et l'enseignement par l'aspect ». Documents pour l'histoire du français langue étrangère ou seconde, 11, 3-13.

REINFREID, Marcus (1999). « Le mouvement réformiste et la méthode directe en Allemagne :

développement, fondement théorique, variations méthodologiques ». Documents pour l'histoire du français langue étrangère ou seconde, 23, 204-226.

\section{NOTES}

1. Cette réforme très importante au niveau du conflit idéologique qui secoue l'enseignement grec est initiée par le gouvernement provisoire d'Elefthérios Vénizélos, installé à Thessalonique (Journal Officiel (désormais J0), no 96/A, 11-7-1917), ratifié par la suite par le Parlement Hellénique

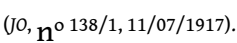

2. Toujours sous le gouvernement d'Elefthérios Vénizélos l'enseignement s'organise en deux cycles, une école primaire de six ans, désormais

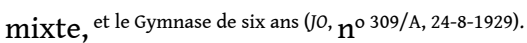

3. Fondée en 1899 à Athènes elle reste en activité jusqu'en 1950.

4. Il faudrait noter que cette année, 1907, marque une étape significative dans l'histoire des manuels car l'État institue le monopole de leur production et de leur diffusion. Par l'intermédiaire des concours lancés tous les quatre ans il contrôle tant le contenu que la disposition des matières, voire leur fabrication $\left(J 0, \mathrm{n}_{\mathrm{O}} 60,4-4-1907\right)$ (Koulouri \& Venturas : 12). Il y avait un manuel unique par matière et par classe.

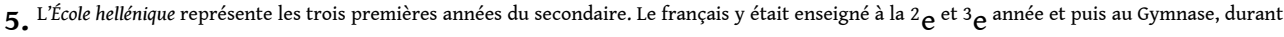
quatre ans. À partir de 1929, on adopte — on l'a dit - une nouvelle répartition : six ans pour l'école primaire et six ans pour le Gymnase.

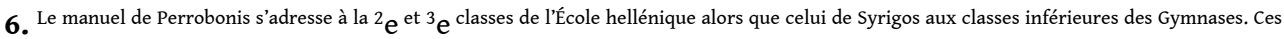

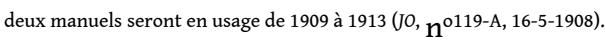

7. Émile Delapalme (1793-1868). Magistrat et membre du Conseil de l'instruction publique (1864), il a publié une série de manuels pour l'instruction des masses populaires, parmi lesquels le Premier livre de l'enfance, ou Exercices de lecture et leçons de morale à l'usage des écoles primaires (1849), plusieurs fois réimprimé.

8. P. J. Stahl est le nom de plume de l'éditeur Pierre-Jules Hetzel (1814-1886).

9. Jean Calvet (1874-1965), professeur à l'Institut Catholique de Paris, est l'auteur de plusieurs manuels scolaires. 


\section{RÉSUMÉS}

Les modifications considérables entreprises dans le domaine de l'enseignement en Grèce dans les premières décennies $\mathrm{du} \mathrm{XX}^{\mathrm{e}}$ siècle, se répercutent aussi dans le domaine de l'enseignement $\mathrm{du}$ français qui s'adapte ainsi au gré d'une législation qui définit la politique éducative et éditoriale. Dans ce cadre sont publiés des manuels inspirés de la méthode directe qui se servent de l'image comme support de l'enseignement. Notre étude se focalise sur l'analyse du rôle de l'image dans les représentations de la culture française véhiculées dans la Collection enfantine de Théodore Kyprios, publiée en Grèce dans les années 1930. Cette série de manuels constitue un tournant dans la conception du matériel didactique utilisé à l'époque pour l'enseignement du français langue étrangère : l'image n'est plus utilisée comme un support au texte mais elle est plutôt conçue comme un outil autonome et indispensable pour l'enseignement-apprentissage de la langue étudiée. L'image n'est plus soumise au texte, comme dans la plupart des manuels de l'époque, mais c'est plutôt le texte qui est soumis à l'image. Sous l'influence de la méthode directe, l'image devient le pivot central d'un enseignement qui bannit le recours à la langue maternelle et qui favorise les fonctions d'observation et d'intuition de l'élève. La culture s'introduit progressivement dans la collection des manuels de Kyprios, suivant le schéma suggéré par les programmes officiels, et se fait plus visible dans les manuels complémentaires

During the first decades of the 20th century, the considerable changes in the field of education in Greece had a significant impact on French language teaching, which is thus adapted to the requirements of legislation defining educational and editorial policy. In this context, textbooks inspired by the direct method were published, using images as a visual aid for teaching. Our study focuses on the analysis of the role of the image in the representations of French culture conveyed in Theodore Kyprios' Children's Collection, published in Greece in the 1930s. This series of textbooks constitutes a turning point in the design of the didactic material, as it was used at the time for the teaching of French as a foreign language: the image no longer complements text but is rather an autonomous and essential tool for language teaching and acquisition. The image is no longer subject to text, as in most manuals of the time, but rather it is the text that is subject to the image. Under the influence of the direct method, the image becomes the central pivot of a teaching that prohibits the use of the mother tongue and promotes the student's observation and intuition. Culture is gradually being introduced into the Kyprios textbook collection, following the pattern suggested by the official curricula, and is becoming more visible in the complementary textbooks.

\section{INDEX}

Keywords : Greece, 20th century, French language teaching, culture, pictures, textbooks, uses of pictures

Mots-clés : Grèce, XXe siècle, enseignement du français, culture, images, manuels, fonctions des images

\section{AUTEURS}

\section{DESPINA PROVATA}

Université nationale et capodistrienne d'Athènes, dprovata[at]frl.uoa.gr 


\section{MARINA VIHOU}

Université nationale et capodistrienne d'Athènes, mvihou[at]frl.uoa.gr 